\title{
ANGULAR ESTIMATIONS OF CERTAIN ANALYTIC FUNCTIONS
}

NAK EUN CHO AND IN HWA KIM

\begin{abstract}
A bostract. The object of the present paper is to investigate some argument properties of certain analytic functions in the open unit disk. Our result contain some interesting corollaries as the special cases.
\end{abstract}

\section{Introduction}

Let $A$ denote the class of functions of the form

$$
f(z)=z+\sum_{n=2}^{\infty} a_{n} z^{n}
$$

which are analytic in the open unit disk $U=\{z:|z|<1\}$. If $f$ and $g$ are analytic in $U$, we say that $f$ is subordinate to $g$, written $f \prec g$ or $f(z) \prec g(z)$, if $g$ is univalent in $U, f(0)=g(0)$ and $f(U) \subseteq g(U)$. Let $S(a, \alpha)$ denote the subclass of $A$ consisting of functions which satisfy

$$
\left|\frac{z f^{\prime}(z)}{f(z)}-a\right|<a-\alpha(z \in U)
$$

for some $\alpha(0 \leq \alpha<1)$ and $a\left(a>\frac{1+\alpha}{2}\right)$. The class $S(a, \alpha)$ was intrdouced by Sekine and Owa[8]. It is clear that, if a function $f$ belongs to the class $S(a, \alpha)$, then

$$
\left|\arg \frac{z f^{\prime}(z)}{f(z)}\right|<\frac{\pi \beta}{2} \quad(z \in U)
$$

where

$$
\beta=\frac{2}{\pi} \operatorname{Sin}^{-1}\left(\frac{a-\alpha}{a}\right) \quad(0 \leq \alpha<1)
$$

Furthermore, we note that for $\alpha=0$, taking $a \rightarrow \infty$, the class $S(a, \alpha)$ is wellknown class of starlike functions with respect to the orign in $U$.

Received April 9, 1997.

1991 Mathematics Subject Classification. 30C45.

Key words and phrases. subordinate, angular estimations, starlike functions 
The purpose of the present paper is to give some argument properties of analytic functions belonging to $A$. Our results have some intersting corollaries and include several previous results of Sakaguchi[7], Libera[1], Macgregor[2], Miller and Mocanu[3] and Nunokawa[6].

\section{Main results}

To esrablish our main results, we need the following lemmas.

Lemma 1([4]). Let $h \in C$, the class of convex functions in $U$, and let $\lambda(z)$ be annlytic in $U$ with $\operatorname{Rc\lambda }(z) \geq 0$. If $p(z)$ is analytic in $U$ and $p(0)=h(0)$, then

$$
p(z)+\lambda(z) z p^{\prime}(z) \prec h(z)(z \in U)
$$

implies

$$
p(z) \prec h(z)(z \in U)
$$

Lemma $2([5])$. Let $P(z)$ be analytic in $U, p(0)=1, p(z) \neq 0$ in $U$ and suppose that there exists a point $z_{0} \in U$ such that

$$
|\arg p(z)|<\frac{\pi \eta}{2} \text { for }|z|<\left|z_{0}\right|
$$

and

$$
\left|\arg p\left(z_{0}\right)\right|=\frac{\pi \eta}{2}
$$

where $\eta>0$. Then we have

$$
\frac{z_{0} p^{\prime}\left(z_{0}\right)}{p\left(z_{0}\right)}=i k \eta
$$

where

$$
k \geq \frac{1}{2}\left(b+\frac{1}{b}\right) \text { when } \arg p\left(z_{0}\right)=\frac{\pi \eta}{2}
$$

and

where

$$
k \leq-\frac{1}{2}\left(b+\frac{1}{b}\right) \text { when } \arg p\left(z_{0}\right)=-\frac{\pi \eta}{2}
$$

$$
p\left(z_{0}\right)^{\frac{1}{n}}= \pm i b(b>0) .
$$

With the help of Lemma 1 and Lemma 2, we now derive

Teorem 1: Let $f \in A$ and $g \in S(a, \alpha)$. If

$$
\left|\arg \left((1-\gamma) \frac{f(z)}{g(z)}+\gamma \frac{f^{\prime}(z)}{g^{\prime}(z)}-\beta\right)\right|<\frac{\pi \delta}{2}(\gamma \geq 0,0 \leq \beta<1,0<\delta \leq 1)
$$


then

$$
\left|\arg \left(\frac{f(z)}{g(z)}-\beta\right)\right|<\frac{\pi \eta}{2},
$$

where $\eta(0<\eta \leq 1)$ is the solution of the equation

$$
\delta=\eta+\frac{2}{\pi} \operatorname{Tan}^{-1}\left(\frac{\gamma \eta \sin \frac{\pi}{2}(1-t(a, \alpha))}{2 a-\alpha+\gamma \eta \cos \frac{\pi}{2}(1-t(a, \alpha))}\right)
$$

and

$$
t(a, \alpha)=\frac{2}{\pi} \operatorname{Sin}^{-1}\left(\frac{a-\alpha}{a}\right) \quad(0 \leq \alpha<1) .
$$

Proof. Let us put

$$
p(z)=\frac{1}{1-\beta}\left(\frac{f(z)}{g(z)}-\beta\right)
$$

Then $p(z)$ is analytic in $U$ with $p(0)=1$. Differentiating (2.3) logarithmically, we have

$$
\frac{1}{1-\beta}\left(\frac{f^{\prime}(z)}{g^{\prime}(z)}-\beta\right)=p(z)+\frac{g(z)}{z g^{\prime}(z)} z p^{\prime}(z) \text {. }
$$

Hence we obtain

$$
(1-\gamma) \frac{f(z)}{g(z)}+\gamma \frac{f^{\prime}(z)}{g^{\prime}(z)}-\beta=(1-\beta)\left(p(z)+\frac{\gamma g(z)}{z g^{\prime}(z)} z p^{\prime}(z)\right)
$$

Applying the assumption and Lemma 1 with $\lambda(z)=\frac{\gamma g(z)}{z g^{\prime}(z)}$, we see that $p(z) \neq 0$ in $U$.

If there exists a point $z_{0} \in U$ such that

$$
|\arg p(z)|<\frac{\pi \eta}{2} \text { for }|z|<\left|z_{0}\right|
$$

and

then, from Lemma 2, we have

$$
\left|\arg p\left(z_{0}\right)\right|=\frac{\pi \eta}{2}
$$

$$
\frac{z_{0} p^{\prime}\left(z_{0}\right)}{p\left(z_{0}\right)}=i k \eta
$$

where

$$
k \geq \frac{1}{2}\left(b+\frac{1}{b}\right) \text { when } \arg p\left(z_{0}\right)=\frac{\pi \eta}{2}
$$

and

$$
k \leq-\frac{1}{2}\left(b+\frac{1}{b}\right) \text { when } \arg p\left(z_{0}\right)=-\frac{\pi \eta}{2},
$$

where

$$
p\left(z_{0}\right)^{\frac{1}{n}}= \pm i b(b>0) .
$$


Since $g \in S(a, \alpha)$ for $a>\frac{1+\alpha}{2}(0 \leq \alpha<1)$,

$$
\frac{z g^{\prime}(z)}{g(z)}=r e^{i \frac{\pi \phi}{2}}
$$

where

$$
\alpha<r<2 a-\alpha
$$

and

$$
-\frac{2}{\pi} \operatorname{Sin}^{-1}\left(\frac{a-\alpha}{a}\right)<\phi<\frac{2}{\pi} \operatorname{Sin}^{-1}\left(\frac{a-\alpha}{a}\right) \quad(0 \leq \alpha<1) .
$$

Suppose that $p\left(z_{0}\right)^{\frac{1}{\eta}}=i b(b>0)$. Then we have

$$
\begin{aligned}
& \arg \left((1-\gamma) \frac{f\left(z_{0}\right)}{g\left(z_{0}\right)}+\gamma \frac{f^{\prime}\left(z_{0}\right)}{g^{\prime}\left(z_{0}\right)}-\beta\right) \\
= & \arg \left((1-\beta) p\left(z_{0}\right)\left(1+\frac{\gamma g\left(z_{0}\right)}{z_{0} g^{\prime}\left(z_{0}\right)} \frac{z_{0} p^{\prime}\left(z_{0}\right)}{p\left(z_{0}\right)}\right)\right) \\
= & \left.\arg p\left(z_{0}\right)+\arg \left(1+\frac{\gamma g\left(z_{0}\right)}{z_{0} g^{\prime}\left(z_{0}\right)} \frac{z_{0} p^{\prime}\left(z_{0}\right)}{p\left(z_{0}\right)}\right)\right) \\
= & \frac{\pi \eta}{2}+\arg \left(1+\gamma\left(r e^{i \frac{\pi \phi}{2}}\right)^{-1} i \eta k\right) \\
= & \frac{\pi \eta}{2}+\operatorname{Tan}^{-1}\left(\frac{\gamma \eta k \sin \frac{\pi}{2}(1-\phi)}{r+\gamma \eta k \cos \frac{\pi}{2}(1-\phi)}\right) \\
\geq & \frac{\pi \eta}{2}+\operatorname{Tan}^{-1}\left(\frac{\gamma \eta \sin \frac{\pi}{2}(1-t(a, \alpha))}{2 a-\alpha+\gamma \eta \cos \frac{\pi}{2}(1-t(a, \alpha))}\right) \\
= & \frac{\pi \delta}{2},
\end{aligned}
$$

where $t(a, \alpha)$ and $\delta$ are given by (2.2) and (2.1), respectively. This is a contradiction to the assumption of our theorem. have

Next, suppose $p\left(z_{0}\right)^{\frac{1}{\eta}}=-i b(b>0)$. Applying the same methad as the above, we

$$
\begin{aligned}
& \arg \left((1-\gamma) \frac{f\left(z_{0}\right)}{g\left(z_{0}\right)}+\gamma \frac{f^{\prime}\left(z_{0}\right)}{g^{\prime}\left(z_{0}\right)}-\beta\right) \\
\leq & -\frac{\pi \eta}{2}-\operatorname{Tan}^{-1}\left(\frac{\gamma \eta \sin \frac{\pi}{2}(1-t(a, \alpha))}{2 a-\alpha+\gamma \eta \cos \frac{\pi}{2}(1-t(a, \alpha))}\right) \\
= & -\frac{\pi \delta}{2},
\end{aligned}
$$

where $t(a, \alpha)$ and $\delta$ are given by (2.2) and (2.1), respectively, which contradicts the assumption. Therefore we complete the proof of our theorem.

Taking $\gamma=1$ in Theorem 1 , we have 
Corrllary 1. Let $f \in A$ and $g \in S(a, \alpha)$, if

$$
\left|\arg \left(\frac{f^{\prime}(z)}{g^{\prime}(z)}-\beta\right)\right|<\frac{\pi \delta}{2}(0 \leq \beta<1,0<\delta \leq 1)
$$

then

$$
\left|\arg \left(\frac{f(z)}{g(z)}-\beta\right)\right|<\frac{\pi \eta}{2}
$$

where $\eta(0<\eta \leq 1)$ is the solution of the equation

$$
\delta=\eta+\frac{2}{\pi} \operatorname{Tan}^{-1}\left(\frac{\eta \sin \frac{\pi}{2}(1-t(a, \alpha))}{2 a-\alpha+\eta \cos \frac{\pi}{2}(1-t(a, \alpha))}\right)
$$

and $t(a, \alpha)$ is given by (2.2).

Remark 1. Taking $\alpha=0, a \rightarrow \infty, \gamma=1$ and $\delta=1$ is Corollary 1 , we obtain the corresponding results of Sakaguchi[5], Libera[1], MacGregor[2] and Miller and Mocanu[3].

Putting $a=1, \alpha \rightarrow 1, \beta=0$ and $g(z)=z$ in Theorem 1, we get

Corollary 2. Let $f \in A$. If

$$
\left|\arg \left((1-\gamma) \frac{f(z)}{z}+\gamma f^{\prime}(z)\right)\right|<\frac{\pi \delta}{2}(\gamma \geq 0,0<\delta \leq 1),
$$

then

$$
\left|\arg \frac{f(z)}{z}\right|<\frac{\pi \eta}{2}
$$

where $\eta(0<\eta \leq 1)$ is the solution of the equation

$$
\delta=\eta+\frac{2}{\pi} \operatorname{Tan}^{-1} \gamma \eta
$$

Similarly, we have

Theorem 2. Let $f \in A$ and $g \in S(a, \alpha)$. If

$$
\left|\arg \left(\beta-\left((1-\gamma) \frac{f(z)}{g(z)}+\gamma \frac{f^{\prime}(z)}{g^{\prime}(z)}\right)\right)\right|<\frac{\pi \delta}{2}(\gamma \geq 0, \beta>1,0<\delta \leq 1),
$$

then

$$
\left|\arg \left(\beta-\frac{f(z)}{g(z)}\right)\right|<\frac{\pi \eta}{2}
$$

where $\eta(0<\eta \leq 1)$ is the solution of the equaiton (2.1).

Remark 2. Putting $\alpha=0, a \rightarrow \infty$ and $\gamma=1$ in Theorem 1 and Teorem 2, we have the corresponding results of Nunokawa[6].

Next, we prove 
Theorem 3. Let $f \in A$. If

$$
\left|\arg \frac{z f^{\prime}(z)}{f^{1-m}(z) z^{m}}\right|<\frac{\pi \delta}{2}(0<\delta \leq 1, m \in N),
$$

then

$$
\left|\arg \left(\frac{f(z)}{z}\right)^{m}\right|<\frac{\pi \eta}{2}
$$

where $\eta(0<\eta \leq 1)$ is the solution of the equation

$$
\delta=\eta+\frac{2}{\pi} \operatorname{Tan}^{-1} \frac{\eta}{m}
$$

Proof. Let us put

$$
p(z)=\left(\frac{f(z)}{z}\right)^{m}
$$

Then $p(z)$ is analytic in $U$ with $p(0)=1$ and we have

$$
\frac{z f^{\prime}(z)}{f^{1-m}(z) z^{m}}=p(z)+\frac{1}{m} z p^{\prime}(z) \text {. }
$$

By the assumption and Lemma 1 , we can see that $p(z) \neq 0$ in $U$. The remaining part of the proof is similar to that of Theorem 1 and so we omit it.

Finally, we have

Theorem 4. Let $f \in A$. If

$$
\begin{aligned}
& \left|\arg \frac{z f^{\prime}(z)}{f^{1-u}(z) z^{u}}\right|<\frac{\pi \delta}{2}(u>0,0<\delta \leq 1), \\
& \left|\arg \frac{z F^{\prime}(z)}{F^{1-u}(z) z^{u}}\right|<\frac{\pi \eta}{2}
\end{aligned}
$$

where $F$ is given by

$$
F^{u}(z)=\frac{u+c}{z^{c}} \int_{0}^{z} t^{c-1} f^{u}(t) d t \quad(c>0)
$$

with $F(z) \neq 0$ in $U-\{0\}$ and $\eta(0<\eta \leq 1)$ is the solution of the equation

$$
\delta=\eta+\frac{2}{\pi} \operatorname{Tan}^{-1} \frac{\eta}{u+c}
$$

Proof. From the definition of (2.5), we have

$$
u \frac{z F^{\prime}(z)}{F^{1-u}(z)}+c F^{u}(z)=(u+c) f^{u}(z) .
$$




\section{Putting}

$$
p(z)=\frac{T(z)}{S(z)}
$$

where $T(z)=F^{\prime}(z) z^{u+c} /(F(z) / z)^{1-u}$ and $S(z)=z^{u+c}$, we have

$$
\frac{T^{\prime}(z)}{S^{\prime}(z)}=p(z)+\frac{1}{u+c} z p^{\prime}(z)=\frac{z f^{\prime}(z)}{f^{1-u}(z) z^{u}}
$$

Applying Lemma 1 and the proof of Theorem 1, we obtain

$$
\left|\arg \frac{z F^{\prime}(z)}{F^{1-u}(z) z^{u}}\right|<\frac{\pi \eta}{2}
$$

where $\eta(0<\eta \leq 1)$ is the solution of the equation (2.6).

\section{Acknowledgements}

This work was partially supported by Non Directed Research Fund, Korea Research Foundation, 1996 and the Basic Science Research Program, Ministry of Education, Project No. BSRI-97-1440.

\section{References}

[1] R. J. Libera, "Some classes of regular univalent functions," Proc. Amer. Math. Soc., 16(1965), 755-758.

[2] T. H. Macgregor, "A subordination for convex functions of order $\alpha$," J. London Math. Soc., (2) $9(1975), 530-536$.

[3] S. S. Miller and P. T. Mocanu, "Second order differential inequalities in the complex plane," J. Math. Anol. Appl., 65(1978), 299-305.

[4] S. S. Miller and P. T. Mocanu, "Differential subordination and univalent functions," Michigan Math.. J., 28(1981), 157-171.

[5] M. Nunokawa, "On the order of strongly starlikeness of strongly convex functions," Proc. Japan Acad., 69, Ser. A(1993), 234.237.

[6] M. Nunokawa, "On some angular estimates of analytic functions," Math. Japonica, 41(1995), 1-6.

[7] K. Sakaguchi, "On a certain univalent mapping," J. Math. Soc. Japan, 11(1959), 72-75.

[8] T. Sekine and S. Owa, "Certain subclasses of starlike functions of order $\alpha$," PanAmer. Math. J., 5(1995), 95-100.

Department of Applied, Mathematics, Pukyong National University, Pusan 608-737, Korea. 